\title{
Dynamic Universe
}

\section{The first person to carry out a modern survey of the night sky, Fritz Zwicky's astronomical observations led to a new picture of a turbulent Universe that is punctuated by violent events.}

\section{Freeman Dyson}

The Swiss physicist Fritz Zwicky (1898-1973) was responsible, more than anyone else, for a profound change in our view of the astronomical Universe. Before Zwicky, the ancient aristotelian view of the celestial sphere as a region of eternal harmony and tranquillity was still largely intact, and the job of an astronomer was to make accurate maps of an unchanging landscape. After Zwicky, the modern view of the Universe emerged, as a dynamic scene dominated by violent events. The job of an astronomer today is to record and interpret the processes of change.

Zwicky's great work was done in the 1930 s when he was an associate professor of physics at Caltech, the California Institute of Technology. He had been trained at the Swiss Federal Institute of Technology as an X-ray crystallographer and had no official credentials as an astronomer. But he saw an opportunity arising from two unconnected events that happened almost simultaneously.

First, in 1928, the Rockefeller Foundation awarded a large sum of money to Caltech for the construction of a major astronomical observatory. Second, in 1930, the one-armed optical genius Bernhard Schmidt in Hamburg invented a telescope with a revolutionary new design, which allowed high-resolution photography over a wide field of view. Zwicky's astronomer friend Walter Baade happened to be a personal friend of Bernhard Schmidt and saw the original Schmidt telescope in action in Hamburg. Zwicky heard what the telescope could do and grabbed the opportunity. He persuaded Caltech to buy an 18-inch Schmidt camera and install it at the new observatory on Palomar Mountain. He made sure that he would have fulltime use of the camera - at that time the only wide-field camera in the world at a site with good astronomical seeing.

Zwicky understood that to see rare, violent and short-lived events in the Universe, he had to photograph large areas of sky repeatedly. With his little Schmidt camera, he had a unique opportunity to photograph the entire northern sky over and over again. With a single assistant to help him, he continued for four years to survey the northern sky. Two major discoveries that emerged from his survey were supernovae and dark matter. Zwicky observed 20 supernovae, a large enough sample to allow him to classify them into several types and infer their different modes of origin. His discovery of dark matter came from studying the motions of individual galaxies in rich clusters of galaxies, and from calculating that the visible mass in the clusters was insufficient by a large factor to cause the observed motions.

flict with his colleagues at Caltech. They considered him crazy and he considered them stupid. He never became an accepted member of the astronomical community, but followed his own path. During the Second World War he was director of research at the Aerojet Corporation, which developed rockets for the military. At the same time he organized the Committee for Aid to War-stricken Scientific Libraries. This collected massive quantities of scientific books and journals and distributed them to libraries that had been disrupted or destroyed during the war. He founded the committee in 1941 and ran it with his habitual enthusiasm and efficiency. For this work he received the Medal of Freedom from President Harry Truman in 1949.

Besides his revolutionary work as an observer and organizer, Zwicky made revolutionary contributions to theoretical astronomy. He published papers about neutron stars, supernovae, black holes and gravitational lenses, long before these subjects became fashionable. His thinking was based on a personal philosophy which he called the 'morphological approach'. The idea is that you first make a complete list of all

In his summary of the survey, Zwicky proudly asserted: ${ }^{\alpha}$ for the construction of the 18-inch Schmidt telescope, its housing, a full-size objective prism, a small remuneration for my assistant, and the operational costs for the whole project during ten years, only about $\$ 50,000$ dollars were expended. This probably represents the highest efficiency, as measured in results achieved per dollar invested, of any telescope presently in use, and perhaps of any ever built, with the exception of Galileïs little refractor."

Zwicky's sky survey set the pattern for many later surveys done with bigger instruments and greater investments of manpower and money. The newest sky survey Pan-STARRS, due to begin in 2006, will follow Zwicky's example in emphasizing rapid and repeated coverage of the sky. It should discover a wealth of short-lived phenomena at all distances, from nearEarth asteroids to optical afterglows of gamma-ray bursts in remote galaxies.

Zwicky's radical ideas and pugnacious personality brought him into frequent con- possible solutions to a problem, and then choose the least unlikely solution for further investigation. By following this approach, you have a good chance of finding new solutions that other people overlooked.

Zwicky applied the morphological approach both to theoretical and practical problems; it was this approach that led him to become the first modern astronomer, with a new and dynamic view of the Universe. In his book Discovery, Invention, Research, Through the Morphological Approach, he describes successful application of the morphological approach to a multitude of problems arising in pure mathematics, automobile design and university administration, as well as in rocket propulsion and astronomy. Unfortunately, the approach does not seem to work so well if your name is not Fritz Zwicky.

Freem an Dyson is at the Institute for Advanced Study, Einstein Drive, Princeton, New Jersey 08540, USA. 